\title{
Study on $Z_{c s}$ and excited $B_{s}^{0}$ states in the chiral quark model
}

\author{
Xiaoyun Chen $\odot,{ }^{1, *}$ Yue Tan, ${ }^{2, \dagger}$ and Yuan Chen ${ }^{3, *}$ \\ ${ }^{1}$ College of Science, Jinling Institute of Technology, Nanjing 211169, People's Republic of China \\ ${ }^{2}$ Department of Physics, Nanjing Normal University, Nanjing 210023, People's Republic of China \\ ${ }^{3}$ Key Laboratory of Radio Frequency and Micro-Nano Electronics of Jiangsu Province, \\ Nanjing 210023, People's Republic of China
}

(Received 19 March 2021; accepted 22 June 2021; published 30 July 2021)

\begin{abstract}
Stimulated by the newly observed charged hidden-charm state $Z_{c s}(3985)^{-}$by BESIII Collaboration, $Z_{c s}(4000)^{+}, Z_{c s}(4220)^{+}$and the excited $B_{s}^{0}$ states by LHCb Collaboration, a full calculation including masses, decay widths, and the inner structures of the states has emerged in the chiral quark model. For $Z_{c s}$ states, we assign quantum numbers $I\left(J^{P}\right)=\frac{1}{2}\left(1^{+}\right)$and quark composition $c \bar{c} s \bar{u}$ according to the experiment. For $B_{s}^{0}$ states, systematically, investigations are performed with $I\left(J^{P}\right)=0\left(0^{+}\right), 0\left(1^{+}\right), 0\left(2^{+}\right)$ in both two-body $b \bar{s}$ and four-body $b \bar{s} q \bar{q}(q=u$ or $d$ ) systems. Each tetraquark calculation takes all structures including meson-meson, diquark-antidiquark, and all possible color configurations into account. Among the numerical techniques to solve the two-body and four-body Schrödinger equation, the spatial wave functions are expanded in series of Gaussian basis functions for high precision, which is the way Gaussian expansion method (GEM) so called. Our results indicate that the low-lying states of the fourquark system are all higher than the corresponding thresholds either for $c \bar{c} s \bar{u}$ or for $b \bar{s} q \bar{q}$ systems. With the help of the real scaling method, we found two molecular resonance states with masses of 4023 and $4042 \mathrm{MeV}$ for the $c \bar{c} s \bar{u}$ system. The state $c \bar{c} s \bar{u}(4042)$ has a close mass and decay width with the recent observed state $Z_{c s}(3985)^{-}$. For the $b \bar{s} q \bar{q}$ system with $J=0$, some resonance states are also found. The newly observed excited $B_{s}^{0}$ states can be accommodated in the chiral quark model as $2 S$ or $1 D$ states, and the mixing with four-quark states also needs to be considered.
\end{abstract}

DOI: $10.1103 /$ PhysRevD.104.014017

\section{INTRODUCTION}

Recently, for the first time, the BESIII Collaboration has reported a structure $Z_{c s}(3985)^{-}$in the $K^{+}$recoil-mass spectrum near the $D_{s}^{-} D^{* 0}$ and $D_{s}^{*-} D^{0}$ mass thresholds in the process of $e^{+} e^{-} \rightarrow K^{+}\left(D_{s}^{-} D^{* 0}+D_{s}^{*-} D^{0}\right)$ at the center-of-mass energy $\sqrt{s}=4.681 \mathrm{GeV}$, with the mass and narrow decay width [1],

$$
\begin{aligned}
M_{Z_{c s}} & =\left(3982_{-2.6}^{+1.8} \pm 2.1\right) \mathrm{MeV}, \\
\Gamma_{Z_{c s}} & =\left(12.8_{-4.4}^{+5.3} \pm 3.0\right) \mathrm{MeV},
\end{aligned}
$$

and the significance was estimated to be $5.3 \sigma$. Soon afterward, a significant state $Z_{c s}(4000)^{+}$, with a mass of $4003 \pm 6_{-14}^{+4} \mathrm{MeV}$, a width of $131 \pm 15 \pm 26 \mathrm{MeV}$, and

\footnotetext{
*xychen@jit.edu.cn

†181001003@njnu.edu.cn

*cy1208@nuaa.edu.cn
}

Published by the American Physical Society under the terms of the Creative Commons Attribution 4.0 International license. Further distribution of this work must maintain attribution to the author(s) and the published article's title, journal citation, and DOI. Funded by SCOAP ${ }^{3}$. spin parity $J^{P}=1^{+}$, was observed by the $\mathrm{LHCb}$ Collaboration, including another exotic state $Z_{c s}(4220)^{+}$ [2]. The discovery of the charged heavy quarkoniumlike structures with strangeness could shed light on the properties of the charged exotic $Z$ states reported before [3].

Besides $Z_{c s}$ states, two excited $B_{s}^{0}$ states have been observed in the $B^{+} K^{-}$mass spectrum in a sample of proton-proton collisions at center-of-mass energies of 7, 8, and $13 \mathrm{TeV}$ very recently by the LHCb Collaboration [4]. The masses and widths of the two states are determined to be

$$
\begin{gathered}
M_{1}=6063.5 \pm 1.2(\text { stat }) \pm 0.8(\text { syst }) \mathrm{MeV}, \\
\Gamma_{1}=26 \pm 4(\text { stat }) \pm 4(\text { syst }) \mathrm{MeV}, \\
M_{2}=6114.5 \pm 3(\text { stat }) \pm 5(\text { syst }) \mathrm{MeV}, \\
\Gamma_{2}=66 \pm 18 \text { (stat) } \pm 21 \text { (syst) } \mathrm{MeV} .
\end{gathered}
$$

For $Z_{c s}$, it is classified into the exotic state as the strange partner of $Z_{c}(3900)$ and has intensively attracted more attention and investigations theoretically within a very short time [5-15]. These explanations basically cover various exotic hadron configurations. One feature of the $Z_{c s}$ is that 
its mass is on the verge of the $\bar{D}_{s} D^{*}$ or $\bar{D}_{s}^{*} D$ threshold, so a molecular resonance is suggested. For example, Lu Meng et al. obtained the mass and width of $Z_{c s}$ in good agreement with the experimental results by considering the coupledchannel effect and strongly supported the $Z_{c s}$ states as the $U / V$-spin partner states of the charged $Z_{c}(3900)$ [5]. In the chiral effective field theory up to the next-to-leading order, $Z_{c s}$ also was regarded as the partner of the $Z_{c}(3900)$ in the SU(3) flavor symmetry and the $\bar{D}_{s} D^{*} / D_{s}^{*} D$ molecular resonance [6]. In the QCD sum rule, $Z_{c s}$ can be well defined as a diquark-antidiquark candidate with quark content $\bar{c} c u \bar{s}$ [8]. In Ref. [12], two models, the chiral quark model and the quark delocalization color screening model, are applied to investigate the system. They exclude the molecular state explanation of $Z_{c s}(3985)$, and some resonance states are predicted by analyzing the effective potentials for the diquark-antidiquark $\operatorname{cs} \bar{c} \bar{u}$ systems.

On the contrary, J. Ferretti pointed out that the mesonmeson molecular model could not be used to describe heavy-light tetraquarks with non-null strangeness content, and in the case of $c \operatorname{sic} \bar{n}(n=u$ or $d)$ configurations, the compact tetraquark ground-state is about $200 \mathrm{MeV}$ below the lowest energy hadrocharmonium state, $\eta_{c} K$ [13]. Another explanation is that $Z_{c s}$ can be naturally regarded as a reflection structure from a charmed-strange meson $D_{s 2}^{*}(2573)$ by the Lanzhou group [14]. By adopting a one-boson-exchange model and considering the coupledchannel effect, Ref. [10] excluded $Z_{c s}$ as a $D^{* 0} D_{s}^{-}$/ $D^{0} D_{s}^{*-} / D^{* 0} D_{s}^{*-}$ resonance. In Ref. [15], the authors try to explain the $Z_{c s}(3985)$ using the chiral constituent quark model in a coupled-channel calculation. In the model, both the $Z_{c s}(3985)$ and the predicted $Z_{c s}(4110)$ are not resonances but a virtual state. As more and more exotic states named as $X Y Z$ have been observed in different experiments, their structures are still inexplicable and controversial theoretically. Investigating charged charmoniumlike states can extend our knowledge of hadrons and our understanding of the nature of strong interaction. So, we believe that the study of the newly observed $Z_{c s}$ states in the chiral quark model can provide some useful information on exotic hadrons. In present work, for $Z_{c s}$, the quantum number is assigned as $I\left(J^{P}\right)=\frac{1}{2}\left(1^{+}\right)$, and the quark composition is $c \bar{c} s \bar{u}$.

Now let us turn to the newly observed excited $B_{s}^{0}$ states. In the past few years, many experiment collaborations such as CDF, D0, and LHCb have made contributions to find the radial and orbital excitations of the bottom and bottomstrange meson families. More and more higher excitations emerged in experiments [16-19]. Therewith, many theoretical studies of the bottom and bottom-strange mesons follow close on another [20-25]. The mass spectrum and strong decay patterns are studied most in the conventional two-body quark-antiquark system, which can describe the ground states very well but has poor understanding for higher excitations of bottom and bottom-strange mesons.
Studying the $B$ and $B_{s}$ mesons will help us not only understand of excited mesons but also put the discovered excited charm and chram-strange mesons into the larger context, since the present situation of experimental exploration of bottom and bottom-strange states is very similar to that of $D$ and $D_{s}$ states in 2003 [26-32]. With new observation of the excited $B_{s}^{0}$ states by the $\mathrm{LHCb}$ Collaboration [4], now it is a good time to carry out a comprehensive theoretical study on higher bottom-strange mesons. In this work, all possible quantum numbers with $I\left(J^{P}\right)=0\left(0^{+}\right), 0\left(1^{+}\right), 0\left(2^{+}\right)$are studied for $B_{s}^{0}$ states. Considering the possible limitation of quark-antidiquark system in the conventional quark model in describing the higher excitations and the possible production of quarkantiquark pair in the vacuum, we obtain the masses of $B_{s}^{0}$ states in two-body quark-antidiquark system and fourbody $b \bar{s} q \bar{q}(q=u$ or $d)$ system, respectively. $b \bar{s} s \bar{s}$ is not included here because of its high energy.

Each tetraquark calculation takes into account the mixing of structures, such as meson-meson and diquarkantidiquark structure, along with all possible color, spin configurations. In the meantime, in order to find possible stable resonance states, high-precision computing way Gaussian expansion method (GEM) [33] and a useful stabilization real scaling method are both employed $[34,35]$ in our calculations.

The paper is arranged as follows. Theoretical framework including the chiral quark model, the wave functions of $Z_{c s}$ and $B_{s}^{0}$, along with GEM are introduced in Sec. II. In Sec. III, the numerical results and discussion are presented. A short summary is given in Sec. IV.

\section{THEORETICAL FRAMEWORK}

\section{A. Chiral quark model}

The review of the chiral quark model and GEM was introduced in Refs. [36-38], and here they will be introduced briefly, and we mainly focus on the relevant features of $Z_{c s}$ and $B_{s}^{0}$ states.

The Hamiltonian of the chiral quark model can be written as follows for the four-body system:

$$
\begin{aligned}
H= & \sum_{i=1}^{4} m_{i}+\frac{p_{12}^{2}}{2 \mu_{12}}+\frac{p_{34}^{2}}{2 \mu_{34}}+\frac{p_{1234}^{2}}{2 \mu_{1234}} \\
& +\sum_{i<j=1}^{4}\left[V_{i j}^{C}+V_{i j}^{G}+\sum_{\chi=\pi, K, \eta} V_{i j}^{\chi}+V_{i j}^{\sigma}\right] .
\end{aligned}
$$

The potential energy $V_{i j}^{C, G, \chi, \sigma}$ represents the confinement, one-gluon-exchange (OGE), Goldstone boson exchange, and $\sigma$ exchange, respectively. The detailed forms can be referred to Eq. (13) in Ref. [36], which are omitted here for space saving. All the model parameters are determined by 
TABLE I. Model parameters, determined by fitting the meson spectrum.

\begin{tabular}{llr}
\hline \hline Quark masses & $m_{u}=m_{d}$ & 313 \\
$(\mathrm{MeV})$ & $m_{s}$ & 536 \\
& $m_{c}$ & 1728 \\
& $m_{b}$ & 5112 \\
Goldstone bosons & $m_{\pi}$ & 0.70 \\
$\left(\mathrm{fm}^{-1} \sim 200 \mathrm{MeV}\right)$ & $m_{\sigma}$ & 3.42 \\
& $m_{\eta}$ & 2.77 \\
& $m_{K}$ & 2.51 \\
& $\Lambda_{\pi}=\Lambda_{\sigma}$ & 4.2 \\
& $\Lambda_{\eta}=\Lambda_{K}$ & 5.2 \\
& $g_{c h}^{2} /(4 \pi)$ & 0.54 \\
& $\theta_{p}\left({ }^{\circ}\right)$ & -15 \\
Confinement & $a_{c}\left(\mathrm{MeV} \mathrm{fm}^{-2}\right)$ & 101 \\
& $\Delta(\mathrm{MeV})$ & -78.3 \\
OGE & $\alpha_{0}$ & 3.67 \\
& $\left.\Lambda_{0}(\mathrm{fm})^{-1}\right)$ & 0.033 \\
& $\mu_{0}(\mathrm{MeV})$ & 36.98 \\
& $s_{0}(\mathrm{MeV})$ & 28.17 \\
\hline \hline
\end{tabular}

fitting the meson spectrum, from light to heavy; and the resulting values are listed in Table I.

It is to be noted that only $V^{\chi=\eta}$ of Goldstone boson exchange plays a role between the $u$ and $s$ quarks, and for the $u$ and $\bar{u}$ interacting quark-pair, not only does $V^{\chi=\pi, \eta}$ work, but also $V^{\sigma}$ works; for other quark pairs such as $(Q, u),(Q, s),(Q, Q)(Q=c, b)$, only $V_{i j}^{C, G}$ is considered without Goldstone bosons and $\sigma$ exchange.

\section{B. Wave functions}

There are three quark configurations for the $Z_{c s}$ and $b \bar{s} q \bar{q}$ system: two meson-meson structures and one diquark-antidiquark structure, which are shown in Fig. 1. We denote $\alpha$ and $\beta$ as the spin-up and spin-down states of

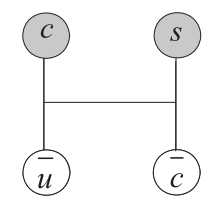

(a)

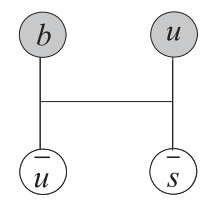

(a')

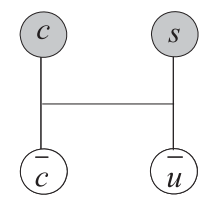

(b)

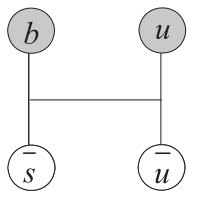

(b')

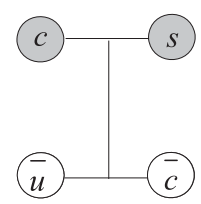

(c)

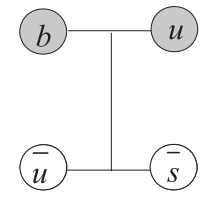

(c')
FIG. 1. Structures of $Z_{c s}$ and $b \bar{s} q \bar{q}$ system, $(a) /\left(a^{\prime}\right)$ and $(b) /\left(b^{\prime}\right)$ represent two meson-meson structures, and $(c) /\left(c^{\prime}\right)$ represents diquark-antidiquark structure. quarks and the spin wave functions for the two-quark system take

$\chi_{11}=\alpha \alpha, \quad \chi_{10}=\frac{1}{\sqrt{2}}(\alpha \beta+\beta \alpha), \quad \chi_{1-1}=\beta \beta$,

$\chi_{00}=\frac{1}{\sqrt{2}}(\alpha \beta-\beta \alpha)$

then a total of six wave functions of the four-body system are obtained easily and are shown in Table II (the first column). The subscript $S M_{S}$ of $\chi$ represents the total spin and the third projection of total spin of four-quark system, with $S=0,1,2$, and only one component $\left(M_{S}=S\right)$ is shown for a given total spin $S$. For the flavor part, the flavor wave functions (two meson-meson structures plus one diquark-antidiquark structure) are also tabulated in Table II (the second column). The wave functions $\chi^{f 1}, \chi^{f 2}$, $\chi^{f 3}$ correspond to the picture $(a),(b),(c)$ in Fig. 1 for $Z_{c s}$, respectively, and the $\chi^{f 4}, \chi^{f 5}, \chi^{f 6}$ are the wave functions of pictures $\left(a^{\prime}\right),\left(b^{\prime}\right),\left(c^{\prime}\right)$ for the $b \bar{s} q \bar{q}$ system. For the color part, there are four wave functions in total (the third column in Table II): $\chi^{c 1}$ color singlet-singlet $(1 \otimes 1)$ and $\chi^{c 2}$ color octet-octet $(8 \otimes 8)$ for meson-meson structure and $\chi^{c 3}$ color antitriplet-triplet $(\overline{3} \otimes 3)$ and $\chi^{c 4}$ sextet-antisextet $(6 \otimes \overline{6})$ for diquark-antidiquark structure.

So, we can get all allowed spin $\otimes$ flavor $\otimes$ color channels of the $Z_{c s}$ and $b \bar{s} q \bar{q}$ system by taking mesonmeson structures, diquark-antidiquark structure, along with all kinds of color spin configurations into account, and these are shown in Table III.

Next, let us discuss the orbital wave functions for the four-body system. They can be obtained by coupling the orbital wave function for each relative motion of the system,

$$
\Psi_{L}^{M_{L}}=\left[\left[\Psi_{l_{1}}\left(\mathbf{r}_{12}\right) \Psi_{l_{2}}\left(\mathbf{r}_{34}\right)\right]_{l_{12}} \Psi_{L_{r}}\left(\mathbf{r}_{1234}\right)\right]_{L}^{M_{L}},
$$

where $l_{1}$ and $l_{2}$ are the angular momentum of two subclusters, respectively. $\Psi_{L_{r}}\left(\mathbf{r}_{1234}\right)$ is the wave function of the relative motion between two subclusters with orbital angular momentum $L_{r} . L$ is the total orbital angular momentum of four-quark states. Because of the positive parity $\left(P=(-1)^{l_{1}+l_{2}+L_{r}}=+\right)$ for $Z_{c s}$ and $b \bar{s} q \bar{q}$, it is natural to assume that all the orbital angular momenta are zeros. With the help of the GEM, the spatial wave functions are expanded in series of Gaussian basis functions,

$$
\begin{gathered}
\Psi_{l}^{m}(\mathbf{r})=\sum_{n=1}^{n_{\max }} c_{n} \psi_{n l m}^{G}(\mathbf{r}), \\
\psi_{n l m}^{G}(\mathbf{r})=N_{n l} r^{l} e^{-\nu_{n} r^{2}} Y_{l m}(\hat{\mathbf{r}}),
\end{gathered}
$$

where $N_{n l}$ are normalization constants, 
TABLE II. The wave functions of spin, flavor, color part for $Z_{c s}$ and $b \bar{s} q \bar{q}$ system by considering all kinds of quark structures.

\begin{tabular}{|c|c|c|}
\hline Spin & Flavor & Color \\
\hline$\chi_{00}^{\sigma 1}=\chi_{00} \chi_{00}$ & $\chi^{f 1}=c \bar{u} s \bar{c}$ & $\chi^{c 1}=\frac{1}{3}(\bar{r} r+\bar{g} g+\bar{b} b)(\bar{r} r+\bar{g} g+\bar{b} b)$ \\
\hline$\chi_{00}^{\sigma 2}=\sqrt{\frac{1}{3}}\left(\chi_{11} \chi_{1-1}-\chi_{10} \chi_{10}+\chi_{1-1} \chi_{11}\right)$ & $\chi^{f 2}=c \bar{c} s \bar{u}$ & $\begin{aligned} \chi^{c 2}= & \frac{\sqrt{2}}{12}(3 \bar{b} r \bar{r} b+3 \bar{g} r \bar{r} g+3 \bar{b} g \bar{g} b+3 \bar{g} b \bar{b} g+3 \bar{r} g \bar{g} r+3 \bar{r} b \bar{b} r \\
& +2 \bar{r} r \bar{r} r+2 \bar{g} g \bar{g} g+2 \bar{b} b \bar{b} b-\bar{r} r \bar{g} g-\bar{g} g \bar{r} r-\bar{b} b \bar{g} g-\bar{b} b \bar{r} r \\
& -\bar{g} g \bar{b} b-\bar{r} r \bar{b} b)\end{aligned}$ \\
\hline$\chi_{11}^{\sigma 3}=\chi_{00} \chi_{11}$ & $\chi^{f 3}=\operatorname{cs} \bar{u} \bar{c}$ & $\begin{aligned} \chi^{c 3}= & \frac{\sqrt{3}}{6}(r g \bar{r} \bar{g}-r g \bar{g} \bar{r}+g r \bar{g} \bar{r}-g r \bar{r} \bar{g}+r b \bar{r} \bar{b}-r b \bar{b} \bar{r} \\
& +b r \bar{b} \bar{r}-b r \bar{r} \bar{b}+g b \bar{g} \bar{b}-g b \bar{b} \bar{g}+b g \bar{b} \bar{g}-b g \bar{g} \bar{b})\end{aligned}$ \\
\hline$\chi_{11}^{\sigma 4}=\chi_{11} \chi_{00}$ & $\chi^{f 4}=\frac{1}{2}(b \bar{d} d \bar{s}+b \bar{u} u \bar{s})$ & $\begin{aligned} \chi^{c 4}= & \frac{\sqrt{6}}{12}(2 r r \bar{r} \bar{r}+2 g g \bar{g} \bar{g}+2 b b \bar{b} \bar{b}+r g \bar{r} \bar{g} \\
& +r g \bar{g} \bar{r}+g r \bar{g} \bar{r}+g r \bar{r} \bar{g}+r b \bar{r} \bar{b}+r b \bar{b} \bar{r} \\
& +b r \bar{b} \bar{r}+b r \bar{r} \bar{b}+g b \bar{g} \bar{b}+g b \bar{b} \bar{g}+b g \bar{b} \bar{g}+b g \bar{g} \bar{b})\end{aligned}$ \\
\hline$\chi_{11}^{\sigma 5}=\frac{1}{\sqrt{2}}\left(\chi_{11} \chi_{10}-\chi_{10} \chi_{11}\right)$ & $\chi^{f 5}=-\frac{1}{2}(b \bar{s} u \bar{u}+b \bar{s} d \bar{d})$ & \\
\hline$\chi_{22}^{\sigma 6}=\chi_{11} \chi_{11}$ & $\chi^{f 6}=-\frac{1}{2}(b u \bar{u} \bar{s}+b d \bar{d} \bar{s})$ & \\
\hline
\end{tabular}

$$
N_{n l}=\left[\frac{2^{l+2}\left(2 \nu_{n}\right)^{l+\frac{3}{2}}}{\sqrt{\pi}(2 l+1)}\right]^{\frac{1}{2}} .
$$

$c_{n}$ are the variational parameters, which are determined dynamically. The Gaussian size parameters are chosen according to the following geometric progression:

$\nu_{n}=\frac{1}{r_{n}^{2}}, \quad r_{n}=r_{1} a^{n-1}, \quad a=\left(\frac{r_{n_{\max }}}{r_{1}}\right)^{\frac{1}{n_{\max }-1}}$.

This procedure enables optimization of the expansion using just a small number of Gaussians. Finally, the complete channel wave function $\Psi_{I J, i, j, k}^{M_{I} M_{J}}$ for the four-quark system is obtained by coupling the orbital and spin, flavor, color wave functions get in Table III:

$$
\begin{aligned}
\Psi_{I J, i, j, k}^{M_{I} M_{J}} & =\mathcal{A}\left[\Psi_{L}^{M_{L}} \chi_{S M_{S}}^{\sigma i}\right]_{J}^{M_{J}} \chi^{f j} \chi^{c k} \\
(i & =1 \sim 6 ; j=1 \sim 6 ; k=1 \sim 4),
\end{aligned}
$$

where $\mathcal{A}$ is the antisymmetrization operator, for the $Z_{c s}$ and $b \bar{s} q \bar{q}$ system, $\mathcal{A}=1$. At last, the eigenvalues of the four-quark system are obtained by solving the Schrödinger equation

$$
H \Psi_{I J}^{M_{I} M_{J}}=E^{I J} \Psi_{I J}^{M_{I} M_{J}}
$$

To obtain stable results in our work, the Gaussian width and Gaussian number of each inner cluster take $r_{1}=0.1 \mathrm{fm}$, $r_{n}=2 \mathrm{fm}, n=12$. For the relative motion between two subclusters, $r_{1}=0.1 \mathrm{fm}, r_{n}=6 \mathrm{fm}, n=7$.

\section{CALCULATIONS AND ANALYSIS}

In the present work, we calculated the mass spectrum of newly observed $Z_{c s}$ and excited $B_{s}^{0}$ states in the chiral

TABLE III. Allowed channels for the $Z_{c s}$ and $b \bar{s} q \bar{q}$ system are showed. The third row Structure $(a),(b),(c)$ represents the three structures for $Z_{c s}$ in Fig. 1. $\left(a^{\prime}\right),\left(b^{\prime}\right),\left(c^{\prime}\right)$ represents the three structures for $b \bar{s} q \bar{q}$ system in Fig 1 . For saving context space, we give abbreviations for channels, e.g., for meson-meson structure [picture $(a)$ ] of $Z_{c s},{ }^{\prime} 311^{\prime}$ represents the third spin wave function $\chi_{11}^{\sigma 3} \otimes$ the first flavor wave function $\chi^{f 1} \otimes$ the first color wave function $\chi^{c 1} .{ }^{\prime} 312^{\prime}$ represents the third spin wave function $\chi_{11}^{\sigma 3} \otimes$ the first flavor wave function $\chi^{f 1} \otimes$ the second color wave function $\chi^{c 2}$. The corresponding wave functions can refer to Table II. The rest of the channels can be read in the same manner. The last row gives the total numbers of channels by considering meson-meson structures, diquark-antidiquark structure, and all kinds of color spin configurations for the $Z_{c s}$ and $b \bar{s} q \bar{q}$ system with quantum numbers $0\left(0^{+}\right), 0\left(1^{+}\right), 0\left(2^{+}\right)$.

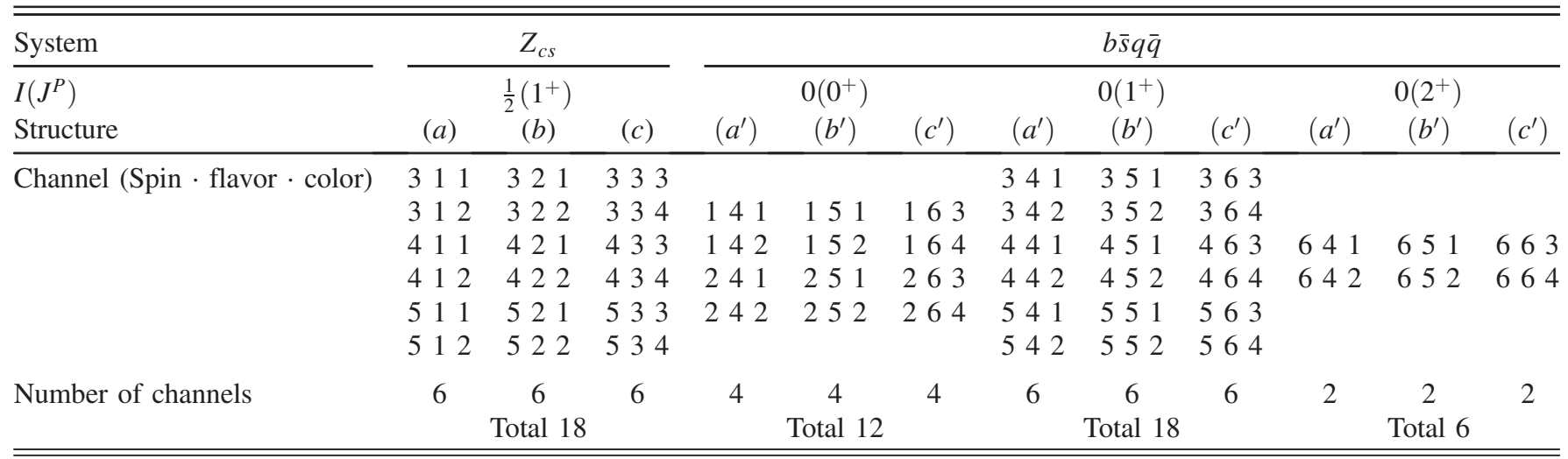


TABLE IV. The mass spectra of $b \bar{s}$ meson families in the chiral quark model in comparison with Ref. [20] and experimental data [39] (unit: MeV).

\begin{tabular}{lccc}
\hline \hline System & $I\left(J^{P}\right)$ & Ref. [20] & Experiment [39] \\
\hline $1^{1} S_{0}$ & 5367.4 & 5390 & $5366.84 \pm 0.15$ \\
$1^{3} S_{1}$ & 5410.2 & 5447 & $5415.8 \pm 1.5$ \\
$2^{1} S_{0}$ & 6017.3 & 5985 & \\
$2^{3} S_{1}$ & 6057.2 & 6013 & \\
$1^{3} P_{0}$ & 5749.2 & 5830 & \\
$1^{3} P_{1}$ & 5779.3 & 5859 & $5828.65 \pm 0.24$ \\
$1^{3} P_{2}$ & 5812.0 & 5875 & $5839.92 \pm 0.14$ \\
$1^{1} P_{1}$ & 5797.6 & 5858 & \\
$2^{3} P_{0}$ & 6345.9 & 6279 & \\
$2^{3} P_{1}$ & 6381.9 & 6291 & \\
$2^{3} P_{2}$ & 6422.9 & 6295 & \\
$2^{1} P_{1}$ & 6403.9 & 6284 & \\
$1^{3} D_{1}$ & 6179.3 & 6181 & \\
$1^{3} D_{2}$ & 6145.3 & 6185 & \\
$1^{3} D_{3}$ & 6094.2 & 6178 & \\
$1^{1} D_{2}$ & 6128.2 & 6180 & \\
$2^{3} D_{1}$ & 6778.1 & 6542 & \\
$2^{3} D_{2}$ & 6743.9 & 6542 & \\
$2^{3} D_{3}$ & 6692.9 & 6534 & \\
$2^{1} D_{2}$ & 6726.8 & 6536 & \\
\hline \hline
\end{tabular}

quark model. For the excited $B_{s}^{0}$ states, we first treat them as ordinary quark-antiquark states. Using the model parameters given in Table I, the convergent results of the $b \bar{s}$ mass spectrum up to the second $D$-wave states in the chiral quark model are obtained and shown in Table IV, where the experimental data are also listed for comparison. Until now, there have been only very limited experimental values on the low-lying bottom-strange mesons, which are called $B_{s}^{0}(5366), B_{s}^{*}(5415), B_{s 1}(5830)^{0}$, and $B_{s 2}^{*}(5840)^{0}$ [39]. In Table IV, we can see that the $2 S$ and $1 D$ states have masses between 6000 and $6200 \mathrm{MeV}$, so it is possible that the newly observed excited $B_{s}^{0}$ are $2 S$ or $1 D$ states of $b \bar{s}$. However, for the excitation energy as high as $700 \mathrm{MeV}$, the excitation of the light quark-antiquark pair from the vaccuum is highly favored. So, considering the excited $B_{s}^{0}$ states as the four-quark $b \bar{s} q \bar{q}(q=u$ or $d)$ system is also necessary. In the following, the four-quark $b \bar{s} q \bar{q}$ $(q=u$ or $d)$ system with quantum numbers $I\left(J^{P}\right)=$ $0\left(0^{+}\right), 0\left(1^{+}\right), 0\left(2^{+}\right)$is investigated.

For $Z_{c s}$, the minimal quark component should be $c \bar{c} s \bar{u}$ rather than a pure $c \bar{c}$ since it is observed as a charged particle with strangeness. Both of $Z_{c s}$ and $b \bar{s} q \bar{q}$ states have two kinds of meson-meson structures and one diquark-antidiquark structure, which are shown in Fig. 1. Along with all possible color and spin configurations, we take all kinds of structures into account. Table $\mathrm{V}$ gives the mass spectra of some relevant quark-antiquark mesons in the present work in the chiral quark model. From the table, we can see that the chiral quark model is very successful in describing the meson spectra. And the mass spectra of $Z_{c s}$ and $b \bar{s} q \bar{q}$ system are demonstrated in Table VI.

From the table, for both the $Z_{c s}$ and $b \bar{s} q \bar{q}$ systems, we can easily find that the low-lying energies in diquarkantidiquark are all much larger than those in meson-meson structures. All of them are higher than the lowest theoretical thresholds. Besides, the effects of the structures mixing seem to be tiny for the ground state energy. The coupling energies $E_{c c}$ are a little higher than the relevant thresholds. So, we cannot find the bound states of the $c \bar{c} s \bar{u}$ and $b \bar{s} q \bar{q}$ tetraquark in the chiral quark model.

Because the colorful clusters cannot fall apart directly, there may exist resonances even with the higher energies. Using the stabilization method (real scaling method), we try to find possible resonance for $c \bar{c} s \bar{u}$ and $b \bar{s} q \bar{q}$ system. To realize the real scaling method here, we multiply the Gaussian size parameters $r_{n}$ in Eq. (9) by a factor $\alpha$,

TABLE V. The mass spectra of some relevant mesons in present work in the chiral quark model, compared with the experimental data [39] (unit: MeV). $q$ represents the $u$ or $d$ quark. (Expt: experiment.).

\begin{tabular}{lcccccccccccc}
\hline \hline$n^{2 S+1} L_{J}$ & $E\left(D^{(*)}\right)$ & Expt & $E\left(D_{s}^{* *}\right)$ & Expt & $E\left(B^{(*)}\right)$ & Expt & $E\left(K^{(*)}\right)$ & Expt & $E(c \bar{c})$ & Expt & $E(q \bar{q})$ & Expt \\
\hline $1^{1} S_{0}$ & 1862.5 & $D(1869)$ & 1952.5 & $D_{s}^{ \pm}(1968)$ & 5280.8 & $B(5279)$ & 493.9 & $K(493)$ & 3097.3 & $\eta_{c}(1 S)(2983)$ & 669.2 & $\eta(547)$ \\
$2^{1} S_{0}$ & 2721.2 & & 2665.3 & & 6090.2 & & 1573.8 & & 3592.1 & $\eta_{c}(2 S)(3639)$ & 1762.2 & \\
$1^{3} S_{1}$ & 1980.5 & $D^{*}(2006)$ & 2079.9 & $D_{s}^{*}(2112)$ & 5319.5 & $B^{*}(5325)$ & 913.5 & $K^{*}(892)$ & 3161.2 & $J / \psi(3096)$ & 701.6 & $\omega(782)$ \\
$2^{3} S_{1}$ & 2829.6 & & 2777.4 & $D_{s 1}^{*}(2700)$ & 6127.7 & & 1887.7 & & 3650.4 & $\psi(2 S)(3686)$ & 1799.2 & \\
$1^{3} D_{1}$ & 3017.6 & & 2872.7 & & 6358.1 & & 1984.9 & & 3706.9 & $\psi(3770)$ & 1954.2 \\
$2^{3} D_{1}$ & 3823.5 & & 3523.1 & & 7122.4 & & 2917.3 & & 4156.6 & & 2998.3 \\
$1^{3} P_{0}$ & 2351.5 & $D_{0}^{*}(2400)$ & 2380.0 & $D_{s 0}^{*}(2317)$ & 5749.7 & & 1149.0 & $K_{0}^{*}(1430)$ & 3420.4 & $\chi_{c 0}(1 P)(3414)$ & \\
$2^{3} P_{0}$ & 3162.3 & & 3028.2 & & 6515.0 & & 2109.6 & & 3869.7 & $\chi_{c 0}(2 P)(3915)$ & \\
$1^{1} P_{1}$ & 2447.9 & & 2478.1 & $D_{s 1}(2460)$ & 5783.4 & $B_{1}(5721)$ & 1400.0 & $K_{1}(1400)$ & 3455.1 & $h_{c}(1 P)(3525)$ & \\
$2^{1} P_{1}$ & 3258.1 & & 3134.9 & & 6554.6 & & 2327.4 & & 3910.7 & & \\
$1^{3} P_{1}$ & 2417.9 & $D_{1}(2420)$ & 2440.9 & & 5777.1 & & 1315.6 & $K_{1}(1270)$ & 3439.7 & $\chi_{c 1}(1 P)(3510)$ & \\
$2^{3} P_{1}$ & 3228.4 & & 3094.9 & & 6545.3 & & 2258.8 & & 3892.9 & $\chi_{c 1}(2 P)(3872)$ & \\
$1^{3} P_{2}$ & 2474.8 & $D_{2}^{*}(2460)$ & 2511.7 & $D_{s 2}^{*}(2573)$ & 5782.9 & $B_{2}^{*}(5747)$ & 1499.5 & $K_{2}^{*}(1430)$ & 3469.6 & $\chi_{c 2}(1 P)(3556)$ & \\
$2^{3} P_{2}$ & 3294.4 & & 3175.9 & & 6559.9 & & 2447.4 & & 3928.0 & $\chi_{c 2}(2 P)(3927)$ & \\
\hline \hline
\end{tabular}


TABLE VI. The low-lying eigenvalues of the $Z_{c s}$ and $b \bar{s} q \bar{q}$ system with allowed quantum numbers. $E(a), E(b), E(c)$ represent the energies in pure meson-meson structures and the pure diquark-antidiquark structure for $Z_{c s}$, corresponding to parts (a), (b), and (c) in Fig. 1. $E(a) \otimes E(c), E(b) \otimes E(c), E(a) \otimes E(b)$ are the energies considering the mixture of one meson-meson structure and one diquark-antidiquark structure, or two meson-meson structures, severally. $E_{c c}$ represents ground state energy for each state after considering the coupling of all possible quark structures, color, and spin channels (refer to Table III). It is the same with $b \bar{s} q \bar{q}$. The last column gives the theoretical lowest thresholds (unit: $\mathrm{MeV}$ ).

\begin{tabular}{cccccccccc}
\hline \hline & & $E(a)$ & $E(b)$ & $E(c)$ & $E(a) \otimes E(c)$ & $E(b) \otimes E(c)$ & $E(a) \otimes E(b)$ & $E_{c c}$ & Lowest thresholds \\
\hline$Z_{c s}$ & \multirow{2}{*}{$\left(1^{+}\right)$} & 3934.5 & 3656.3 & 4247.1 & 3934.5 & 3655.4 & 3934.5 & 3656.2 & $3655.1\left(J / \psi K^{-}\right)$ \\
& & $E\left(a^{\prime}\right)$ & $E\left(b^{\prime}\right)$ & $E\left(c^{\prime}\right)$ & $E\left(a^{\prime}\right) \otimes E\left(c^{\prime}\right)$ & $E\left(b^{\prime}\right) \otimes E\left(c^{\prime}\right)$ & $E\left(a^{\prime}\right) \otimes E\left(b^{\prime}\right)$ & $E_{c c}$ & Lowest thresholds \\
$b \bar{s} q \bar{q}$ & $0\left(0^{+}\right)$ & 5776.6 & 6040.2 & 6283.2 & 5776.5 & 6040.2 & 5776.6 & 5775.3 & $5774.8\left(B^{-} K^{+}\right)$ \\
& $0\left(1^{+}\right)$ & 5815.4 & 6072.6 & 6316.9 & 5815.3 & 6072.6 & 5815.4 & 5814.1 & $5813.5\left(B^{*-} K^{+}\right)$ \\
& $0\left(2^{+}\right)$ & 6234.8 & 6114.9 & 6483.7 & 6234.7 & 6114.9 & 6234.8 & 6114.3 & $6111.7\left(\overline{B_{s}}{ }^{*} \omega\right)$ \\
\hline \hline
\end{tabular}

$r_{n} \rightarrow \alpha r_{n}$ only for the meson-meson structure with the color singlet-singlet configuration. Then, we can locate the resonances of $c \bar{c} s \bar{u}$ and $b \bar{s} q \bar{q}$ system with respect to the scaling factor $\alpha$, which takes the values from 1.0 to 3.5. With the variation of $\alpha$, the scattering states will level off to corresponding thresholds, but a resonance will appear as an

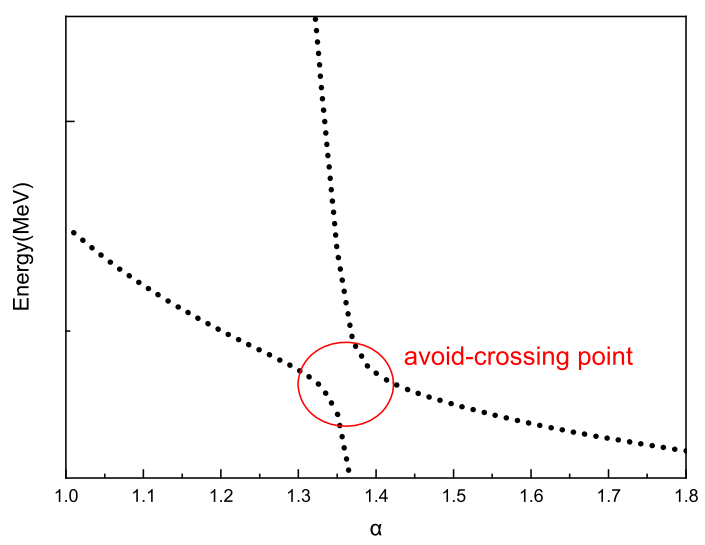

FIG. 2. Stabilization graph for the resonance.

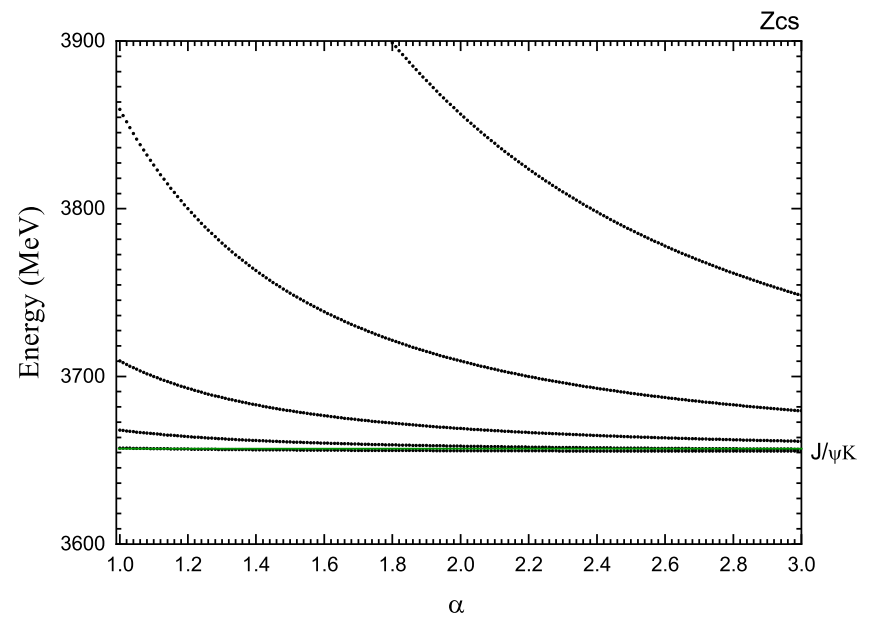

FIG. 3. The stabilization plots of the energies (3600$3900 \mathrm{MeV})$ of $c \bar{c} s \bar{u}$ states for $I\left(J^{P}\right)=\frac{1}{2}\left(1^{+}\right)$with respect to the scaling factor $\alpha$. avoid-crossing structure, which is illustrated in Fig. 2 [34]. The above line represents a scattering state, and it will fall down to the threshold. The down line is the resonance state, which tries to keep stable. The resonance state will interact with the scattering state, which can bring about an avoidcrossing point in Fig. 2. With the increasing of the scaling factor $\alpha$, if the time of repetition of avoid-crossing points is larger than or equals 2, it will be a resonance [34].

To make it clear for the reader, we illustrated the stabilization plots of the energies from 3600 to $4100 \mathrm{MeV}$ for $Z_{c s}$ states, respectively, in Figs. 3-5 [Fig. 3 (3600 3900 MeV), Fig. 4 (3900 4000 MeV), Fig. 5 (4000 4100 MeV)]. In Fig. 3, we see the first green horizontal line, which represents the lowest threshold $J / \psi K(1 \otimes 0 \rightarrow 1)$. In the energy region 3900 to $4000 \mathrm{MeV}$ (Fig. 4), there are two thresholds $D^{* 0} D_{s}^{-}(1 \otimes$ $0 \rightarrow 1)$ and $D^{0} D_{s}^{*}(0 \otimes 1 \rightarrow 1)$. In the higher energy range 4000 to $4100 \mathrm{MeV}$ in Fig. 5, two thresholds $\eta_{c} K^{*}(0 \otimes$ $1 \rightarrow 1)$ and $D^{* 0} D_{s}^{*}(1 \otimes 1 \rightarrow 1)$ appear. Meanwhile, in the figure, we can clearly see the repeated avoid-crossing points which are marked with red circles, and the red

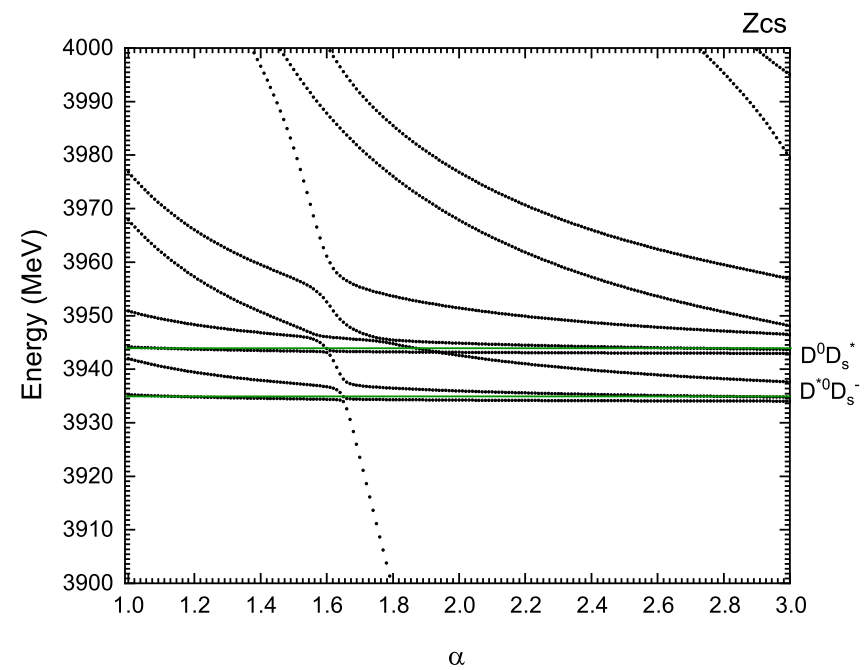

FIG. 4. The stabilization plots of the energies (3900$4000 \mathrm{MeV})$ of $c \bar{c} s \bar{u}$ states for $I\left(J^{P}\right)=\frac{1}{2}\left(1^{+}\right)$with respect to the scaling factor $\alpha$. 


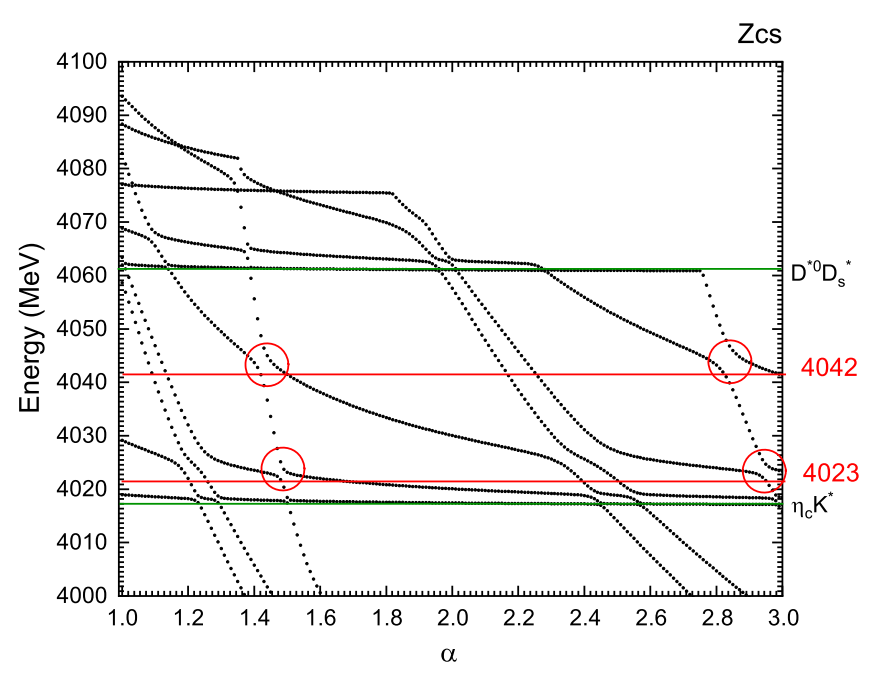

FIG. 5. The stabilization plots of the energies (4000$4100 \mathrm{MeV})$ of $c \bar{c} s \bar{u}$ states for $I\left(J^{P}\right)=\frac{1}{2}\left(1^{+}\right)$with respect to the scaling factor $\alpha$.

horizontal lines are on behalf of two genuine resonance states, with the energy 4023 and $4042 \mathrm{MeV}$. The energies of the resonances are not far from the experimental values of $Z_{c s}(3985)^{-}$observed by BESIII and $Z_{c s}(4000)^{+}$ observed by LHCb. To see the structures of resonances, the percentages of each channel of the resonances are also calculated in Table VII. The results show that the resonances with energies 4023 and $4042 \mathrm{MeV}$ are molecular states, and the dominant components are $D^{*} D_{s}^{*}$.

For the $b \bar{s} q \bar{q}$ system, we show the results with all possible quantum numbers $I\left(J^{P}\right)=0\left(0^{+}\right), 0\left(1^{+}\right)$, and $0\left(2^{+}\right)$in Figs. 6-10. Figures 6-8 represent the $b \bar{s} q \bar{q}$ system for $0\left(0^{+}\right)$. In the energy range 5700 to $6000 \mathrm{MeV}$ (Fig. 6), there is one threshold $B K$, and no resonance is found. Above $6000 \mathrm{MeV}$ in Figs. 7 and 8, we find several resonances, such as 6050, 6078, 6140, 6155, and $6241 \mathrm{MeV}$. Besides these resonance states, we can find that there exist some repetition avoid-crossing points just above and very close to the thresholds line. In our work, we regard them as the threshold effect but resonance states. From Fig. 7, we can see that nearby the energy with $6155 \mathrm{MeV}$, two repetition avoid-crossing points emerge. Similarly, nearby the energy with $6140 \mathrm{MeV}$, we also

TABLE VII. The decay widths and the dominant component of predicted resonances of $c \bar{c} s \bar{u}$ and $b \bar{s} q \bar{q}$ system. (unit: MeV).

\begin{tabular}{lrc}
\hline \hline Resonance state & $\Gamma$ & Dominant component \\
\hline$c \bar{c} s \bar{u}(4023)$ & 3.1 & $D^{*} D_{s}^{*}(89 \%)$ \\
$c \bar{c} s \bar{u}(4042)$ & 13.7 & $D^{*} D_{s}^{*}(90 \%)$ \\
$b \bar{s} q \bar{q}(6050)$ & 7.8 & $B_{s}^{0} \eta(99 \%)$ \\
$b \bar{s} q \bar{q}(6078)$ & 44.1 & $B_{s}^{0} \eta(80 \%)$ \\
$b \bar{s} q \bar{q}(6140)$ & 18.3 & $B_{s}^{*} \omega(80 \%)$ \\
$b \bar{s} q \bar{q}(6155)$ & 8.7 & $B_{s}^{*} \omega(80 \%)$ \\
$b \bar{s} q \bar{q}(6241)$ & 4.1 & $B^{-} K^{+}(70 \%)$ \\
\hline \hline
\end{tabular}

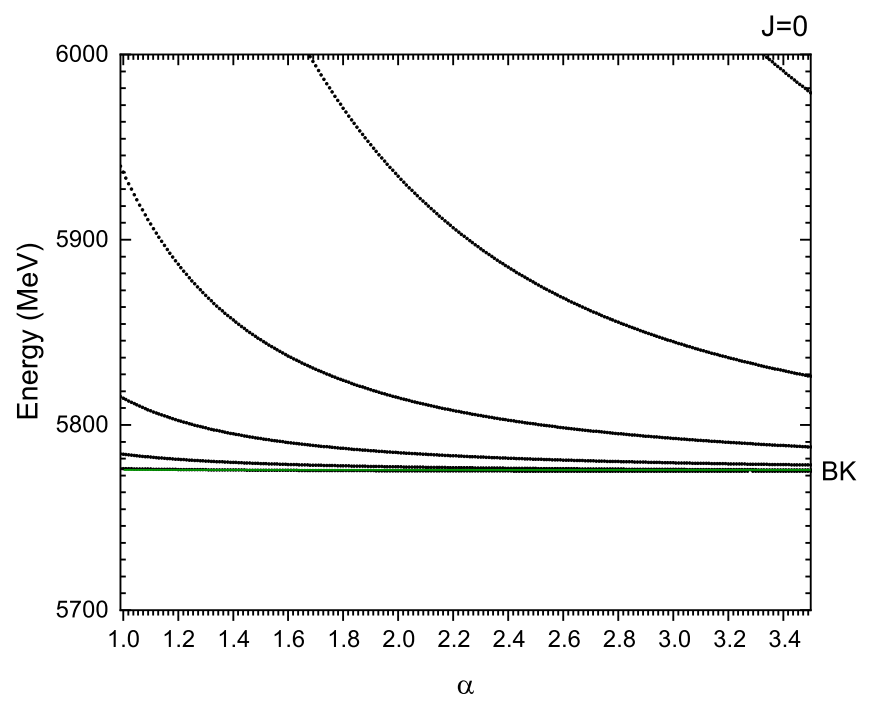

FIG. 6. The stabilization plots of the energies (5700$6000 \mathrm{MeV})$ of $b \bar{s} q \bar{q}$ states for $I\left(J^{P}\right)=0\left(0^{+}\right)$with respect to the scaling factor $\alpha$.

find two repetition avoid-crossing points. At these avoidcrossing points, the line with smaller slope is the resonance state, which tries to keep stable. Otherwise, the line with bigger slope is the scattering state. At the avoid-crossing point, the resonance state and scattering state couples strongest, and that is the reason the avoid-crossing points emerge. So, we can easily find that the first avoid-crossing point respectively for 6155 and $6140 \mathrm{MeV}$ shares the same resonance line (with smaller slope), as well as the second repeated avoid-crossing point for them. So, in our calculations, we just found one resonance with energy 6140 to $6155 \mathrm{MeV}$. Besides, we calculated the proportions of total 12 channels for this resonance, and the results indicate that it has a dominant molecular component $B_{s}^{*} \omega(80 \%)$.

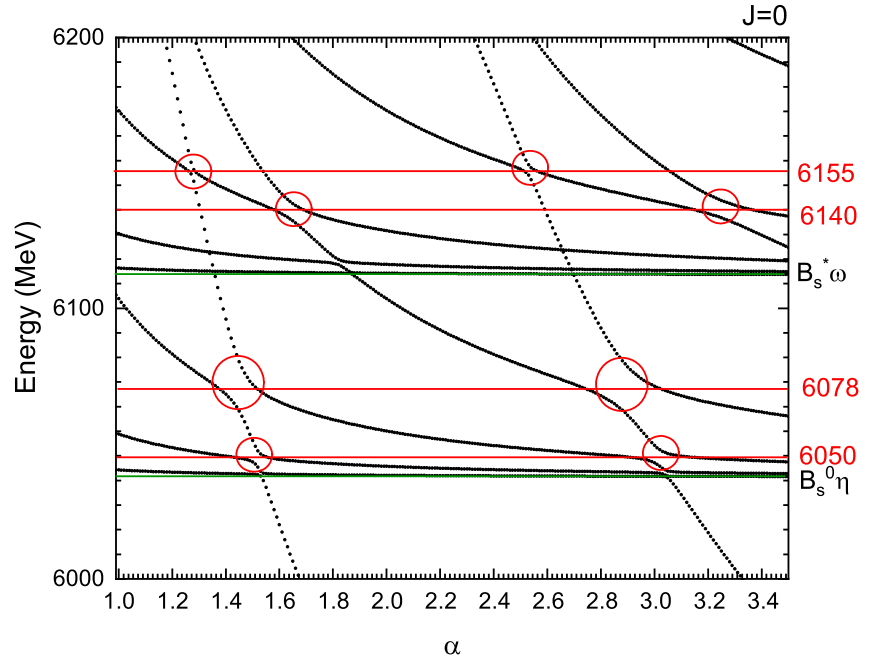

FIG. 7. The stabilization plots of the energies (6000$6200 \mathrm{MeV})$ of $b \bar{s} q \bar{q}$ states for $I\left(J^{P}\right)=0\left(0^{+}\right)$with respect to the scaling factor $\alpha$. 


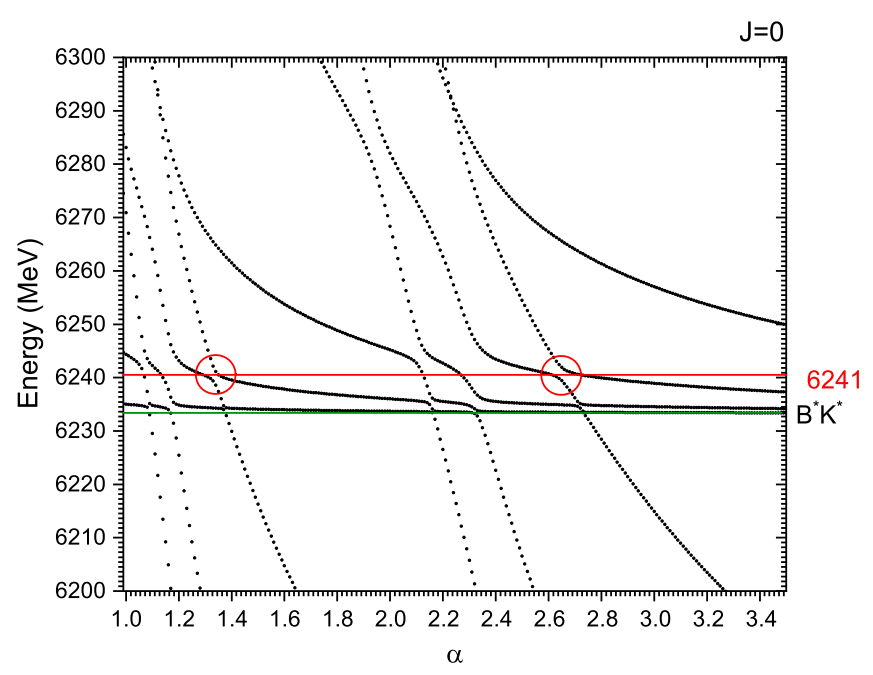

FIG. 8. The stabilization plots of the energies (6200$6300 \mathrm{MeV})$ of $b \bar{s} q \bar{q}$ states for $I\left(J^{P}\right)=0\left(0^{+}\right)$with respect to the scaling factor $\alpha$.

In Table VII, the dominant component of the other resonances of the $b \bar{s} q \bar{q}$ system is demonstrated. The results show that these resonances all have a dominant molecular component. For $0\left(1^{+}\right)$and $0\left(2^{+}\right)$states in Figs. 9 and 10, we cannot find any resonance states in our work.

What is more, we calculated the decay widths of these resonance states using the formula taken from Ref. [34],

$$
\Gamma=4|V(\alpha)| \frac{\sqrt{\left|S_{r}\right|\left|S_{c}\right|}}{\left|S_{c}-S_{r}\right|},
$$

where $V(\alpha)$ is the difference between the two energies at the avoid-crossing point with the same value $\alpha . S_{r}$ and $S_{c}$ are the slopes of scattering line and resonance line, respectively. For each resonance, we get the decay width at the first and the second avoid-crossing points, and we finally give the average decay width of these two values.

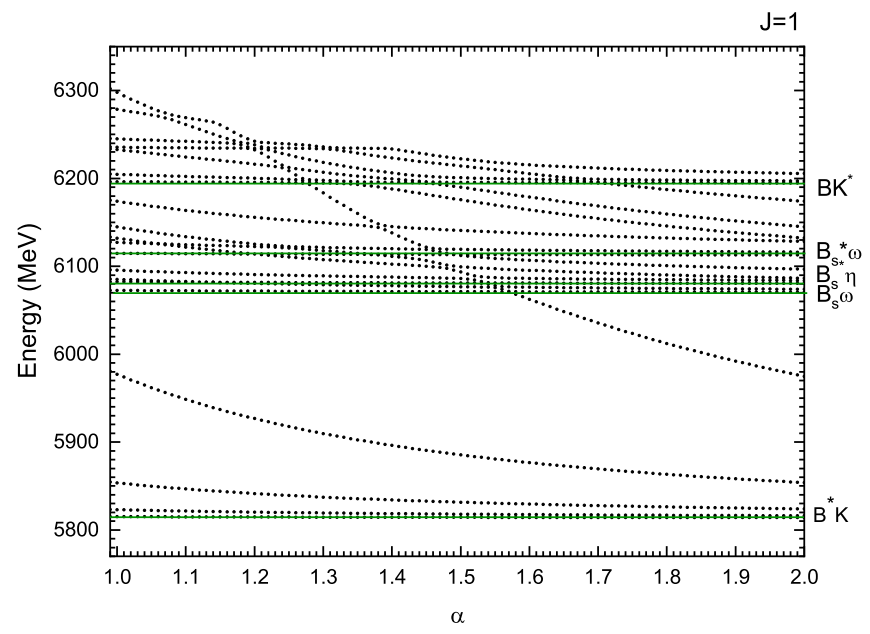

FIG. 9. The stabilization plots of the energies of $b \bar{s} q \bar{q}$ states for $I\left(J^{P}\right)=0\left(1^{+}\right)$with respect to the scaling factor $\alpha$.

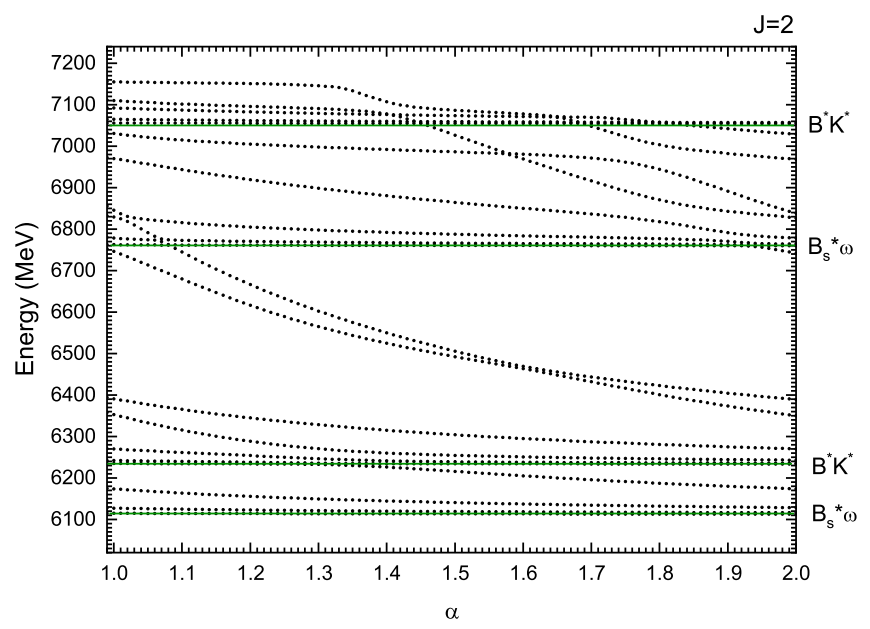

FIG. 10. The stabilization plots of the energies of $b \bar{s} q \bar{q}$ states for $I\left(J^{P}\right)=0\left(2^{+}\right)$with respect to the scaling factor $\alpha$.

The results are shown in Table VII. For the $c \bar{c} s \bar{u}(4042)$ state, the decay width of $13.7 \mathrm{MeV}$ is very consistent with the experimental values of $Z_{c s}(3985)^{-}$, with the decay width of $12.8 \mathrm{MeV}$. Besides, we can see that the mass and decay width of the $b \bar{s} q \bar{q}(6078)$ state are relatively close to the experimental values $M=6063 \mathrm{MeV}$ and $\Gamma=26 \mathrm{MeV}$ by LHCb Collaboration [4]. Combining with the results of the $b \bar{s}$ system, it is possible that the newly observed excited $B_{s}^{0}$ states are mixing states of $b \bar{s}$ and $b \bar{s} q \bar{q}(q=u, d)$. The unquenched quark model should be invoked to study the highly excited mesons.

\section{SUMMARY}

Motivated by the recent experimental information from BESIII and LHCb collaborations, we calculated the masses, decay width, and the inner structures of the $Z_{c s}$ with $I\left(J^{P}\right)=\frac{1}{2}\left(1^{+}\right)$and $B_{s}^{0}$ states with $I\left(J^{P}\right)=$ $0\left(0^{+}\right), 0\left(1^{+}\right), 0\left(2^{+}\right)$in the framework of the chiral quark model using the Gaussian expansion method. Mesonmeson and diquark antidiquark structures and the coupling of them are considered.

For the $Z_{c s}$ state with quark component $c \bar{c} s \bar{u}$, we found that the low-lying eigenvalues are all higher than the corresponding thresholds in either structure, leaving no space for a bound state. But we found two resonances with mass 4023 and $4042 \mathrm{MeV}$ for the $c \bar{c} s \bar{u}$ system with the help of the real scaling method, and the decay widths are 3.1 and $13.7 \mathrm{MeV}$, respectively. The state $c \bar{c} s \bar{u}(4042)$ has a close mass to the recent observed state $Z_{c s}(3985)^{-}$and $Z_{c s}(4000)^{+}$, but the decay width is close to the experimental value of $Z_{c s}(3985)^{-}$and far narrower than the experimental value $Z_{c s}(4000)^{+}$. Besides, we found that the resonances $c \bar{c} s \bar{u}(4023)$ and $c \bar{c} s \bar{u}(4042)$ are molecular states, and the dominant components are $D^{*} D_{s}^{*}$.

To find the excited $B_{s}^{0}$ state observed by the $\mathrm{LHCb}$ Collaboration, we give the mass spectrum in both the two- 
body $b \bar{s}$ system and four-body $b \bar{s} q \bar{q}(q=u$ or $d$ ) system by considering the possible production of quark-antiquark pair in the vacuum. For the quark-antiquark system, the $2 S$ and $1 D$ states have masses close to the newly observed $B_{s}^{0}$, so the chiral quark model can accommodate these excited $B_{s}^{0}$ states. For four-quark system, no bound state is found. However, several molecular resonances have emerged. They have energies 6050, 6078, 6140 6155, and $6241 \mathrm{MeV}$. The decay widths are all relatively narrow.
Comparing with the experimental data, we found that it is also possible to interpret the observed $B_{s}^{0}$ states as fourquark states. Therefore, the better way to investigate the highly excited states is to invoke the unquenched quark model [36], which is our future work.

These possible resonant states should be tested in more precise experimental data in the future, and we need more experimental studies on the dominant decay channels of $Z_{c s}$ and $B_{s}$ to figure out their inner configurations.
[1] M. Ablikim et al. (BESIII Collaboration), Phys. Rev. Lett. 126, 102001 (2021).

[2] R. Aaij et al. (LHCb Collaboration), arXiv:2103.01803.

[3] M. Ablikim et al., Phys. Rev. Lett. 110, 252001 (2013); 111, 242001 (2013); 112, 022001 (2014).

[4] R. Aaij et al. (LHCb Collaboration), arXiv:2010.15931.

[5] L. Meng, B. Wang, and S. L. Zhu, Phys. Rev. D 102, 111502 (R) (2020).

[6] B. Wang, L. Meng, and S. L. Zhu, Phys. Rev. D 103, L021501 (2021).

[7] K. Azizi and N. Er, Eur. Phys. J. C 81, 61 (2021).

[8] J. Y. Süngü, A. Türkan, H. Sundu, and E. V. Veliev, arXiv: 2011.13013.

[9] Q. N. Wang, W. Chen, and H. X. Chen, arXiv:2011.10495.

[10] R. Chen and Q. Huang, Phys. Rev. D 103, 034008 (2021).

[11] Z. Yang, X. Cao, F. K. Guo, J. Nieves, and M. P. Valderrama, Phys. Rev. D 103, 074029 (2021).

[12] X. Jin, X. Liu, Y. Xue, H. Huang, and J. Ping, arXiv: 2011.12230.

[13] J. Ferretti and E. Santopinto, J. High Energy Phys. 04 (2020) 119.

[14] J. Z. Wang, Q. S. Zhou, X. Liu, and T. Matsuki, Eur. Phys. J. C 81, 51 (2021).

[15] P. G. Ortega, D. R. Entem, and F. Fernández, Phys. Lett. B 818, 136382 (2021).

[16] T. A. Aaltonen et al. (CDF Collaboration), Phys. Rev. D 90, 012013 (2014).

[17] T. Aaltonen et al. (CDF Collaboration), Phys. Rev. Lett. 100, 082001 (2008).

[18] V. M. Abazov et al. (D0 Collaboration), Phys. Rev. Lett. 100, 082002 (2008).

[19] R. Aaij et al. (LHCb Collaboration), Phys. Rev. Lett. 110, 151803 (2013).

[20] Y. Sun, Q. T. Song, D. Y. Chen, X. Liu, and S. L. Zhu, Phys. Rev. D 89, 054026 (2014).
[21] L. Y. Xiao and X. H. Zhong, Phys. Rev. D 90, 074029 (2014).

[22] J. B. Liu and M.Z. Yang, Phys. Rev. D 91, 094004 (2015).

[23] S. Godfrey, K. Moats, and E. S. Swanson, Phys. Rev. D 94, 054025 (2016).

[24] Q. F. Lü, T. T. Pan, Y. Y. Wang, E. Wang, and D. M. Li, Phys. Rev. D 94, 074012 (2016).

[25] J. Ferretti and E. Santopinto, Phys. Rev. D 97, 114020 (2018).

[26] B. Aubert et al. (BABAR Collaboration), Phys. Rev. Lett. 90, 242001 (2003).

[27] D. Besson et al. (CLEO Collaboration), Phys. Rev. D 68, 032002 (2003); 75, 119908(E) (2007).

[28] P. Krokovny et al. (Belle Collaboration), Phys. Rev. Lett. 91, 262002 (2003).

[29] A. V. Evdokimov et al. (SELEX Collaboration), Phys. Rev. Lett. 93, 242001 (2004).

[30] B. Aubert et al. (BABAR Collaboration), Phys. Rev. Lett. 97, 222001 (2006).

[31] B. Aubert et al. (BABAR Collaboration), Phys. Rev. D 80, 092003 (2009).

[32] P. del Amo Sanchez et al. (BABAR Collaboration), Phys. Rev. D 82, 111101 (2010).

[33] E. Hiyama, Y. Kino, and M. Kamimura, Prog. Part. Nucl. Phys. 51, 223 (2003).

[34] J. Simon, J. Chem. Phys. 75, 2465 (1981).

[35] Emiko Hiyama, Atsushi Hosaka, Makoto Oka, and JeanMarc Richard, Phys. Rev. C 98, 045208 (2018).

[36] X. Chen, J. Ping, C. D. Roberts, and J. Segovia, Phys. Rev. D 97, 094016 (2018).

[37] X. Chen and J. Ping, Phys. Rev. D 98, 054022 (2018).

[38] X. Chen and J. Ping, Eur. Phys. J. C 76, 351 (2016).

[39] P. A. Zyla et al. (Particle Data Group), Prog. Theor. Exp. Phys. 2020, 083 C01 (2020). 